\title{
Mineraçãåo
}

\section{Aplicação da espectroscopia de reflectância difusa na quantificação dos constituintes}

\author{
Application of diffuse reflectance spectroscopy \\ in the quantification of the constituents of \\ bauxite and iron ore
}

\begin{abstract}
Ana Cláudia Carioca
M.Sc. em Engenharia Mineral, Universidade Federal de Ouro Preto aclaudiacarioca@yahoo.com.br

\section{Geraldo Magela da Costa \\ Departamento de Química, \\ Universidade Federal de Ouro Preto \\ magela@iceb.ufop.br}

\section{Vidal Barrón}

Departamento de Ciências e Recursos

Agrículas e Florestais, Universidad de Córdoba, 14071 Córdoba, Espanha

vidal@uco.es

\section{Cesar Mendonça Ferreira \\ Departamento de Geologia, \\ Universidade Federal de Ouro Preto \\ mendonca.cesar@uol.com.br}

\section{Jose Torrent}

Departamento de Ciências e Recursos

Agrículas e Florestais, Universidad de Córdoba, 14071 Córdoba, Espanha

torrent@uco.es

\section{Resumo}

Esse trabalho teve como objetivo a aplicação da espectrofotometria de reflectância difusa no desenvolvimento de uma metodologia para a identificação e quantificação de minerais presentes na bauxita e nos minérios de ferro. Amostras selecionadas contendo uma variabilidade mineral foram coletadas na região do Quadrilátero Ferrífero e na região de Carajás e os teores dos diferentes minerais foram estimados a partir de uma combinação de métodos convencionais, tais como análise química, difração de raios X, espectroscopia Mössbauer, susceptibilidade magnética e microscopia ótica. Os dados espectrais obtidos na reflectância difusa nas regiões do visível e do infravermelho juntamente com a composição mineralógica foram analisados por algoritmos quimiométricos. A análise de correlação mineralógica apresentou resultados satisfatórios, tendo parâmetros estatísticos médios próximos de $\mathrm{R}^{2}=0,93$, para bauxita, e de $\mathrm{R}^{2}=0,87$, para minério de ferro. Por exemplo, para a goethita, presente no minério de ferro, o $\mathrm{R}^{2}=0,96$; com erro-padrão médio de calibração (RMSEC) $=3,6 \%$, e, para a hematita, presente também no minério de ferro, o $\mathrm{R}^{2}=0,90$ com o $\mathrm{RMSEC}=7,3 \%$. Os resultados sugerem que a espectroscopia de reflectância difusa é uma ferramenta promissora para a quantificação simultânea dos minerais presentes em bauxita e minério de ferro.

Palavras-chave: Reflectância difusa, bauxita, minério de ferro.

\section{Abstract}

This paper describes the application of diffuse reflectance spectroscopy in the development of a methodology for the identification and quantification of minerals in bauxite and iron ores. Selected samples containing a mineral variability were collected from the Iron Quadrangle (Minas Gerais) mines and from the region of Carajás (Pará). The different mineral concentrations were estimated from a combination of conventional methods such as chemical analysis, Mössbauer spectroscopy, Xray diffractions, magnetic susceptibility and optical microscopy. Diffuse reflectance spectra in the visible, near infrared and mid infrared regions were correlated to the mineralogical composition by chemometric algorithms. The mineralogical analysis showed satisfactory correlations, and on the average the statistical parameters were found to be close to $R^{2}=0.93$ for bauxite and $R^{2}=0.87$ for iron ores. Goethite $\left[R^{2}\right.$ $=0.96$, average standard error of validation $(R M S E C)=3.6 \%]$ and hematite $\left(R^{2}=\right.$ 
0.90$, RMSEC $=7.3 \%)$ for iron ore. These results suggest that the diffuse reflectance spectroscopy is a fast and easy method for the simultaneous determination of various minerals present in bauxite and iron ores.

Keywords: Diffuse reflectance, bauxite, iron ore.

\section{Introdução}

Muitos minérios brasileiros apresentam estruturas complexas e muito variadas, devido às diferentes condições de metamorfismo e intemperismo a que foram sujeitos, ou, mesmo, em virtude de sua gênese. Dessa forma, originaram-se minérios com diferentes constituintes mineralógicos, tamanho e morfologia dos cristais, tamanho e morfologia dos poros, formas e superfícies das partículas e cores variadas.

A complexidade dos minérios, tanto sob aspectos físicos, quanto químicos, vem exigindo estudos de caracterização das propriedades dos seus minerais, precedendo a rota de beneficiamento mineral. Tal procedimento é imprescindível, não apenas pelas questões intrínsecas aos minérios, mas pela necessidade de se aperfeiçoar o processo de obtenção do melhor produto ao menor custo, propiciar uma melhor viabilidade econômica e uma menor geração de impacto ambiental (Oliveira \& Neumann, 2009). Por exemplo, os minerais de ferro na bauxita podem influenciar no desempenho do material frente ao processo Bayer, especialmente na sedimentação da lama vermelha. Desta forma, a identificação e a quantificação das fases mineralógicas constituintes da bauxita e dos minérios de ferro representam um assunto de suma importância para a área mineral.

Os principais constituintes minerais

\section{Materiais e métodos}

Amostras sólidas foram obtidas após o processo de cominuição (britagem e moagem). Para as amostras de bauxita, procedeu-se à pulverização em um moinho de bolas, obtendo, no final, uma granulometria abaixo de $0,045 \mathrm{~mm}$ e para as de minério de ferro procedeuse à separação granulométrica, sendo utilizada a amostra passante na peneira de $100 \#(0,150 \mathrm{~mm})$. Foi analisado um total de 97 amostras de bauxita, oriundas do Estado do Pará, e 114 de minério de ferro, das quais 87 amostras foram coletadas na região do Quadrilátero Ferrífero (Minas Gerais) e 27, no Estado do Pará.

Os espectros Mössbauer foram de bauxitas brasileiras são a gibbsita $\left(\mathrm{Al}(\mathrm{OH})_{3}\right)$, goethita $(\alpha-\mathrm{FeOOH})$, hematita $\left(\alpha-\mathrm{Fe}_{2} \mathrm{O}_{3}\right)$, caolinita $\left(\mathrm{Al}_{2} \mathrm{Si}_{2} \mathrm{O}_{5}(\mathrm{OH})_{4}\right)$ e anatásio $\left(\mathrm{TiO}_{2}\right)$. Em jazidas mais antigas, pode-se encontrar, também, a boehmita (AlOOH) (Costa \& Neumann, 2007). Nos minérios de ferro as fases predominantes são a hematita (especularita e martita), goethita, magnetita $\left(\mathrm{Fe}_{3} \mathrm{O}_{4}\right)$ e quartzo $\left(\mathrm{SiO}_{2}\right)$.

A técnica mais utilizada para a identificação mineral é a difração de raios $\mathrm{X}$, porém é raramente utilizada para a quantificação devido a efeitos que interferem na intensidade dos picos, por exemplo, efeitos de textura (orientação preferencial), estresse e tamanho do cristal (Jenkins \& Snyder, 1996). O método de Rietveld é utilizado na difração de raios X para quantificação das fases cristalinas, mas o procedimento não é simples para amostras que apresentam efeitos texturais (Rietveld, 1969; Pöllmann \& Angélica, 2002). Entretanto, da Costa et al. (2002) descreveram um método que utiliza a combinação dos resultados de análises químicas e de difração de raios $\mathrm{X}$ que possibilita a quantificação de algumas fases mineralógicas presentes em minérios de ferro.

A espectroscopia de reflectância difusa, um tipo de análise espectral, era, até há pouco tempo, somente utilizada para a identificação e a quantificação de

coletados em temperatura ambiente com um espectrômetro convencional de 512 canais, na faixa de velocidade de -11 a $11 \mathrm{~mm} / \mathrm{s}$. A calibração da velocidade foi obtida a partir do padrão $\alpha-\mathrm{Fe}$. Os ajustes dos espectros foram realizados computacionalmente com sextetos e/ou dubletos simétricos (Bancroft, 1973). Os teores de cada fase mineralógica contendo ferro foram calculados com o uso das áreas relativas obtidas nos ajustes espectrais e os valores de ferro total foram obtidos por análise química.

Os difratogramas de raios X foram obtidos através de um aparelho Shimadzu XRD 6000, equipado com tubo de Fe e com um monocromador de grafite. minerais em solos e sedimentos (Torrent \& Barrón, 1993; Torrent \& Barrón, 2008). A aplicação dessa técnica espectroscópica, na região do espectro visível $(300$ a 800 $\mathrm{nm}$ ) e do infravermelho próximo (800 a $2500 \mathrm{~nm}$ ), juntamente com análises quimiométricas, vem fornecendo resultados satisfatórios para a caracterização mineral (Ben-Dor \& Banin, 1990; Reeves et al., 1999; Malley et al., 2004; Viscarra-Rossel et al., 2006). Nguyen et al. (1991) realizaram estudos para avaliar a possibilidade de caracterização de silicatos, óxidos/ hidróxidos de ferro e alumínio na região do infravermelho médio (2500 a 25000 $\mathrm{nm})$. No entanto, até o presente momento, esse processo ainda não foi aplicado para a quantificação de minerais em bauxita. A quantificação por espectroscopia de reflectância difusa, na região do visível, das fases presentes em minérios de ferro foi descrita recentemente por da Costa et al (2009).

Portanto, o presente trabalho foi realizado com o objetivo de se investigar a possível aplicação da espectroscopia de reflectância difusa para a identificação e a quantificação das principais fases presentes na bauxita e no minério de ferro. Essa técnica de análise pode fornecer resultados em poucos minutos, tem custo relativamente baixo e não requer preparação da amostra. Os resultados obtidos foram muito promissores, como será demonstrado a seguir.

As varreduras foram feitas entre $7-70^{\circ}$ (2-theta) com velocidade do goniômetro de $2^{\circ}$ por minuto. Foi utilizado o programa JADE para a identificação das fases mineralógicas presente nas amostras.

A microscopia de luz refletida (Leica, modelo MPS30), com uma magnificação de 200 vezes, foi utilizada para a contagem e identificação de, pelo menos, 500 partículas. As percentagens volumétricas foram convertidas em peso, utilizando-se as densidades médias de cada espécie mineral: especularita $=5,2$ g.cm ${ }^{-3} ;$ martita $=3,9$ g.cm ${ }^{-3} ;$ goethita $=$ 3,0 g.cm $\mathrm{cm}^{-3} ;$ magnetita $=5,0$ g.cm $\mathrm{cm}^{-3}$; quart$\mathrm{zo}=2,65 \mathrm{~g} . \mathrm{cm}^{-3}$ (da Costa et al., 2009). As medidas de susceptibilidade 
magnética foram feitas em um equipamento Bartington MS-2B de dupla freqüência (Bartington Instrumentss Ltd., Oxford. Reino Unido). O teor de magnetita foi calculado usando-se um valor de susceptibilidade magnética de $5 \times 10^{-4} \mathrm{~m}^{3} \mathrm{~kg}^{-1}$ (Dearing, 1999).

Os espectros de reflectância difusa foram obtidos em três diferentes equipamentos: 1) espectrofotômetro Cary 5000 Vis-IR (Varian Inc., Palo Alto, CA), equipado com uma esfera de integração de $110 \mathrm{~mm}$, sendo que os valores de reflectância foram adquiridos no intervalo de 300-2500 nm com intervalo de 0,5 $\mathrm{nm}$; 2) espectrofotômetro portátil ASD Inc. LabSpec 5000 (Analytical Spectral Devices, Inc., Boulder, CO), equipado com três detectores dependentes do

\section{Resultados e discussões}

Os minerais predominantes nas amostras de bauxita são a gibbsita, hematita, goethita, caolinita, anatásio e rutilo. Algumas amostras apresentaram, ainda,

\section{Análise espectral}

Os espectros dos padrões de especularita, martita, goethita e quartzo, nas regiões do visível e infravermelho, são mostrados na Figura 1. Observa-se que existem diferenças significativas, tanto nas posições, quanto nas intensidades das bandas, o que possibilita a identificação dessas fases mineralógicas.

Embora exista certa similaridade comprimento de onda e uma sonda modelo A122374, sendo que as medidas foram feitas nos comprimentos de onda entre 350 e $2500 \mathrm{~nm}$ em intervalos de $1 \mathrm{~nm}$; 3) espectrofotômetro Tensor 27 MIR (Bruker, Ettlingen, Alemanha), equipado com uma semi-esfera de reflectância difusa Pike EasiDiff, sendo que os dados espectrais foram coletados entre 4000 a $400 \mathrm{~cm}^{-1}(2500-25000 \mathrm{~nm}) \mathrm{em}$ intervalos de $4 \mathrm{~cm}^{-1}$. Os espectros de reflectância foram utilizados nas análises quimiométricas após a transformação da porcentagem de reflectância em absorbância [ $\log _{10}$ (1/Reflectância)].

Análise de componentes principais (PCA) usando o algoritmo NIPALS (Martens \& Naes, 1989) e análise de regressão dos quadrados mínimos parciais

a boehmita. A mineralogia das amostras de minério de ferro é mais simples, consistindo, basicamente, de hematita (especularita, martita), goethita, magnetita e
(PLSR) (Geladi \& Kowaslski, 1986), tal como implementada no programa Parles (Viscarra-Rossel, 2008), foram utilizadas para correlacionar os espectros com a composição mineral determinada pelas diversas técnicas anteriormente mencionadas. A calibração foi avaliada através do coeficiente de determinação, $\mathrm{R}^{2}$, e do erro de calibração (SEC). O ensaio de validação foi feito usando-se um procedimento de validação cruzada, o que implica a remoção de uma amostra de todo o conjunto e o desenvolvimento de um modelo de calibração com as demais, estabelecendo uma previsão para o valor da amostra removida. Nesse caso, a avaliação do ajuste é dada pelo erro-padrão de predição (SEV) (Shenk $\&$ Westerhaus, 1996).

quartzo. Entretanto algumas amostras apresentaram os minerais caolinita e moscovita. entre os espectros da especularita e martita, existem alguns detalhes diferentes, na região de infravermelho médio (MIR). A intensidade e a largura dos picos são diferentes, sendo os picos da especularita mais bem definidos que os da martita. Além disso, existe um pequeno deslocamento das bandas. No espectro da goethita, na região MIR, observam-se uma banda larga próxima a $3600 \mathrm{~cm}^{-1}$ e um pico próximo a 900 $\mathrm{cm}^{-1}$, devido ao estiramento e à deformação dos grupamentos $-\mathrm{OH}$, respectivamente (Russell \& Fraser 1994). Com relação à região do visível, observa-se a existência de bandas largas, tanto para especularita, quanto para martita e goethita.
Figura 1

Curvas de reflectância difusa de especularita (a), martita (b), goethita (c) e quartzo (d).

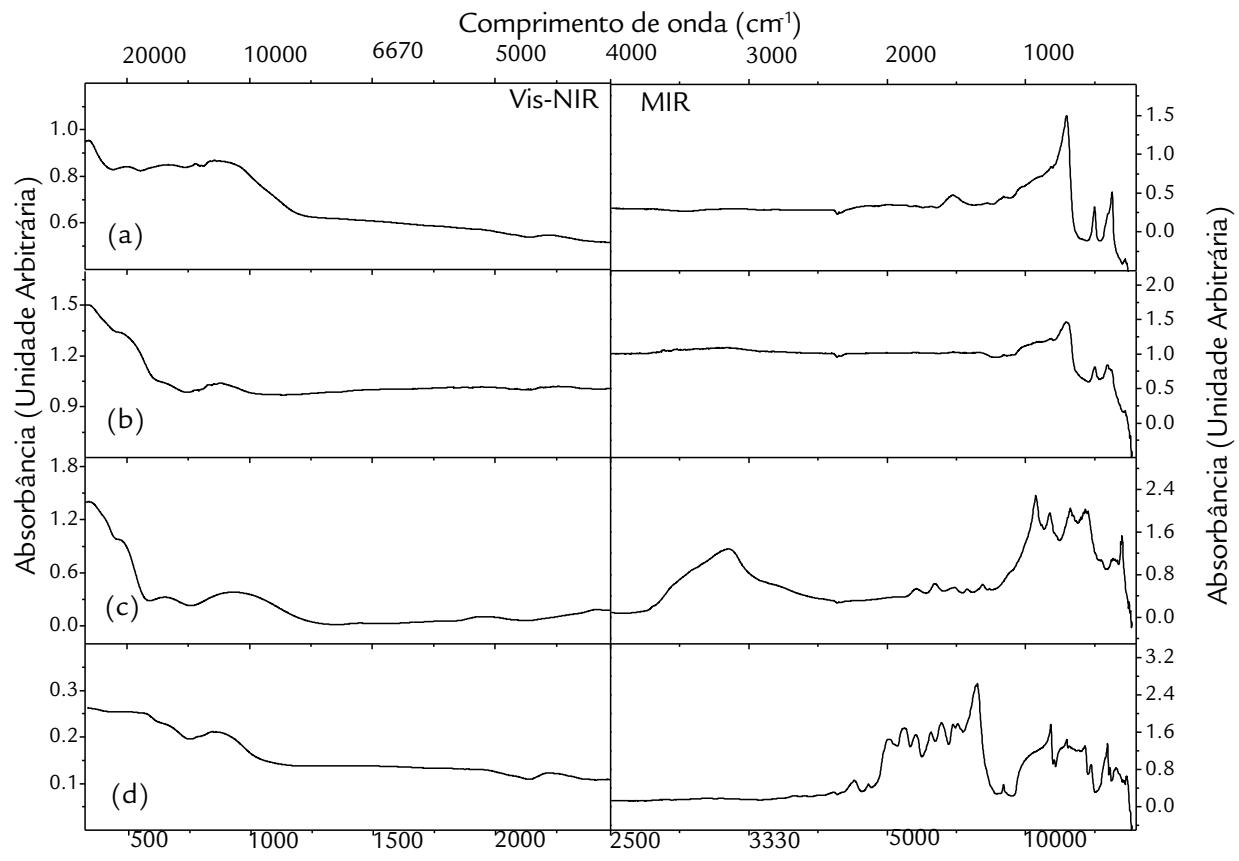

Comprimento de onda $(\mathrm{nm})$ 


\section{Análises quimiométricas}

$\mathrm{Na}$ análise por componentes principais (PCA), um pré-processamento nos dados foi necessário para atribuir pesos equivalentes aos dados espectrais das amostras. A PCA mostrou que com seis componentes principais é possível descrever 99,99\% dos dados, sendo que $98,29 \%$ da variância total foi descrita pela primeira componente principal.

A partir dos dados espectrais obtidos nos equipamentos CARY, ASD e TENSOR, utilizando-se comprimentos de onda nas regiões do visível, infravermelho próximo e infravermelho médio, e no software quimiométrico PARLES, obteve-se um modelo com capacidade

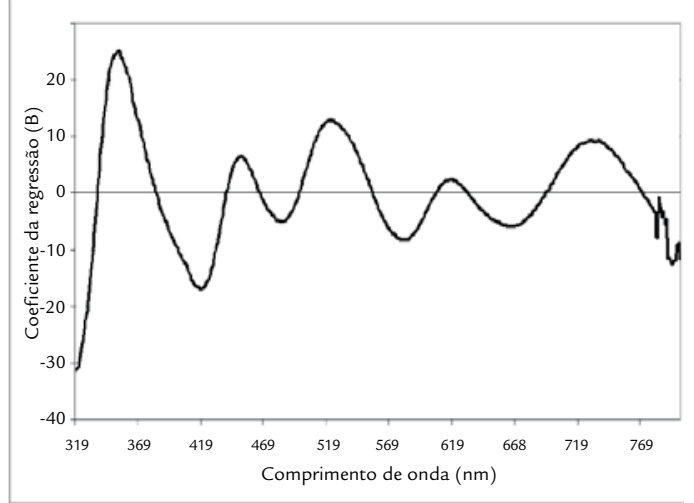

de predizer o conteúdo mineral para uma nova amostra. Um exemplo para a determinação do teor de ferro total, utilizando-se o equipamento CARY 5000, no comprimento de onda entre 319 a 800 nm, está mostrado na Figura 2 e na Equação 1.

O coeficiente $\mathrm{B}_{0}$ é igual a 12, sendo

Figura 2

Representação gráfica do modelo de regressão utilizada para predizer o conteúdo de ferro total.

$$
(\%) \mathrm{Fe}=\mathrm{B}_{0}+\mathrm{B}_{319} *(\log 1 / \mathrm{R})_{319}+\mathrm{B}_{320} *(\log 1 / \mathrm{R})_{320}+\mathrm{B}_{321} *(\log 1 / \mathrm{R})_{321}+\ldots+\mathrm{B}_{800} *(\log 1 / \mathrm{R})_{800}
$$

que 19 e $B_{x}$ são os coeficientes da regressão em comprimentos de onda específicos. Os valores dos coeficientes que são repre-

\section{Correlações}

Os modelos de calibração correlacionando os teores dos minerais medidos por diversas técnicas com as curvas de
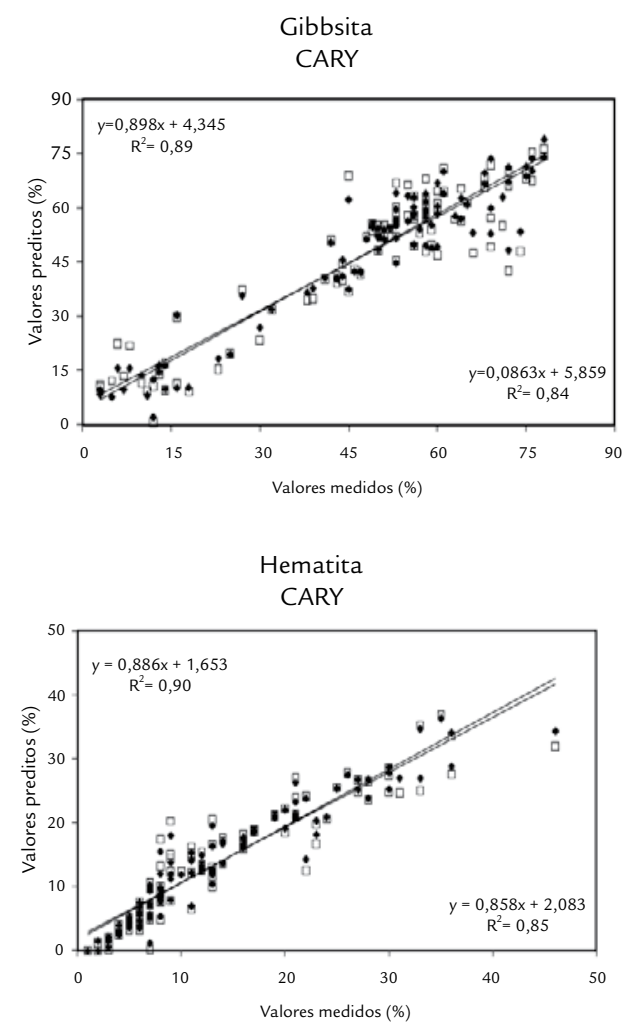

sentados pelos máximos e mínimos na Figura 2 são realmente os comprimentos de onda que mais influenciam na regressão, isto é, são os que mais influenciam nos resultados para os cálculos dos teores dos minerais existentes em novas amostras. reflectância difusa foram obtidos através do programa PARLES pelo método de calibração cruzada um a um. Os melho-
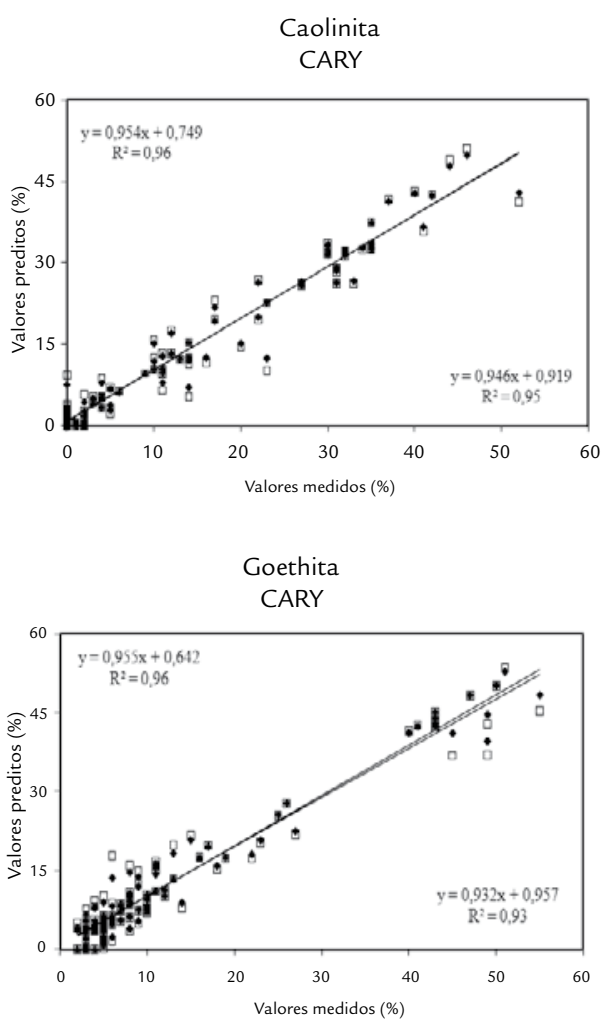

res modelos de calibração obtidos, para alguns minerais estão representados nas Figuras 3 e 4, para bauxita e minério de
Figura 3

Valores preditos de calibração (quadrados preto) e validação (quadrados branco) versus valores medidos dos principais minerais de bauxita.

Medidas realizadas na região Vis-NIR (319 a $2500 \mathrm{~nm}$ ) no equipamento CARY 5000. 
ferro, respectivamente.

Segundo Malley et al. (2004), os valores de calibração para minerais podem ser divididos em quatro categorias. Quando se obtêm valores de calibração acima de $\mathrm{R}_{\mathrm{C}}^{2}>0,95$, casos da goethita e

Figura 4

Valores preditos de calibração (quadrados pretos) e validação (quadrados brancos) versus valores medidos dos principais minerais de minério de ferro.

Foi observado, para alguns minerais, que os melhores modelos de calibração são encontrados, quando se seleciona uma dada região espectral, em comparação com os modelos obtidos, quando se emprega o espectro todo (Spiegelman et

\section{Conclusão}

Os resultados mostraram que a espectroscopia de reflectância difusa, nas regiões do visível, infravermelho próximo e médio, é uma técnica que pode ser usada para a identificação e a quantificação simultânea de várias fases mineralógicas

\section{Agradecimentos}

Esse trabalho foi parcialmente financiado pela Fapemig e CNPq (Brazil)

\section{Referências bibliográficas}

caolinita, considera-se que a calibração foi excelente. Para valores entre $R_{C}^{2}=0,90 \mathrm{a}$ 0,95 , ocorreu uma calibração bem sucedida, por exemplo para hematita (bauxita e minério de ferro) e gibbsita. Para valores de $\mathrm{R}_{\mathrm{C}}^{2}$ entre 0,8 a 0,89 , é considerado que a calibração foi moderadamente bem sucedida, caso do quartzo e da especularita. Para valores no intervalo de $\mathrm{R}_{\mathrm{C}}^{2}=$ 0,7 a 0,8 , considera-se que a calibração é moderadamente útil, como foi o caso da martita.
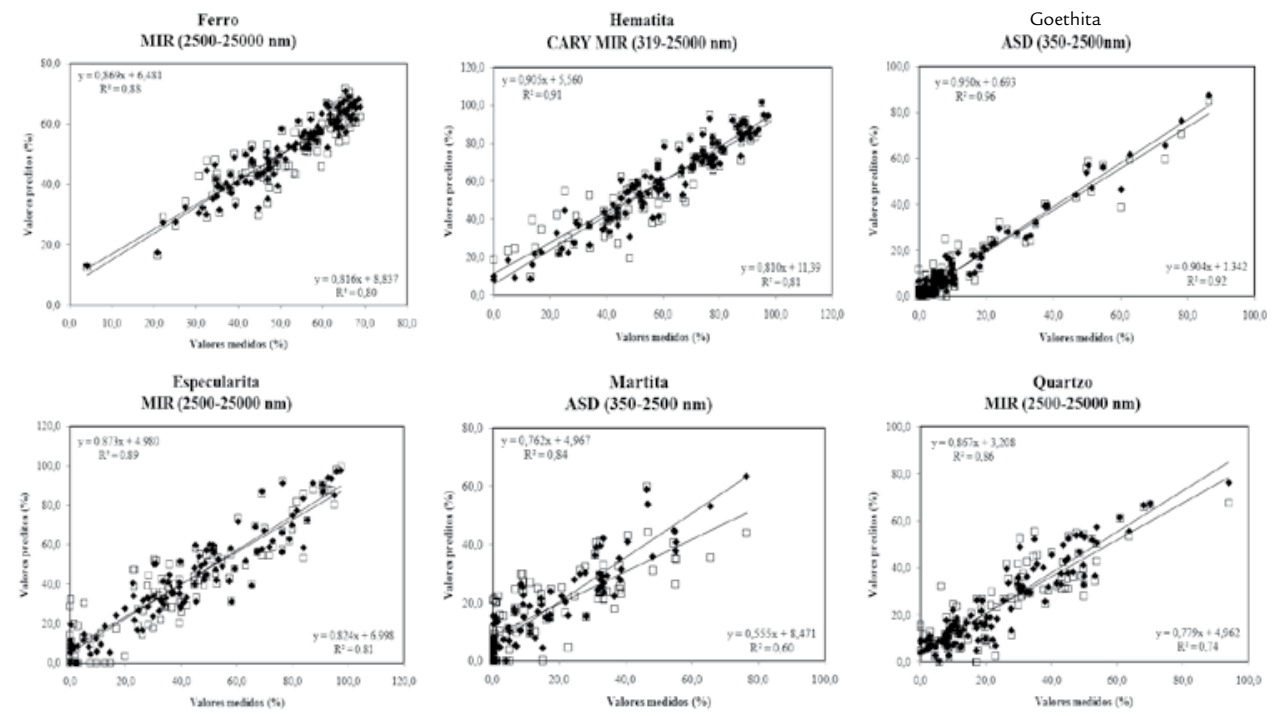

al., 1998). Na região em que os minerais têm uma resposta espectral constituída por bandas largas, a maior resolução é obtida com o aparelho CARY 5000, em comparação com o equipamento portátil ASD. Entretanto o ASD forneceu melho- res correlações e apresenta uma grande vantagem em relação ao CARY 5000, pois é um equipamento portátil e, assim, as análises podem ser realizadas in situ, diretamente no campo e com uma maior rapidez. presentes em amostras de bauxita e de minério de ferro.

Essa técnica permite uma análise rápida (menos de 5 minutos) e de baixo custo, fácil de ser realizada, e que não requer preparação prévia das amostras.
Além disso, é uma técnica não destrutiva e que poderá ser utilizada para a realização de medidas in-situ, com equipamentos similares ao espectrômetro portátil ASD. e pelo Ministerio de Ciencia e Innovación da Espanha (Projecto CGL2010-15067) e FEDER.

BANCROFT, G.M. Mössbauer Spectroscopy: An Introduction for Inorganic Chemists and Geochemists. John Wiley \& Sons, 1973. p. 252

BEN-DOR, E., BANIN, A. Diffuse reflectance spectra of smectite minerals in the near infrared and their relationship to chemical composition. Sciences Geologiques Bulletin, 43, p. 117-128, 1990.

COSTA, L. B. F. da, NEUMAN, R. Quantificação de fases minerais e de amorfas por difração de raios X, método de refinamento de espectros multifasicos total [Método de Rietveld]. JORNADA DE INICIAÇÃO CIENTIFICA- CETEM, 15. Anais... p. 1-7, 2007.

da COSTA, G. M., BARRÓN, V., FERREIRA, C. M., TORRENT, J. The use of diffuse reflectance spetroscopy for the characterization of iron ores. Minerals Engineering, 22, p. 1245-1250, 2009.

da COSTA, G. M., DE RESENDE, V. G., TORÍBIO, N.M., Quantitative phase 
analysis of iron ore concentrates. REM- Revista Escola de Minas, v. 55, n. 4, p. 263266, 2002.

DEARING, J. Environmental Magnetic Susceptibility: using Bartington MS2 System. Kenilworth: Chi Publishing, 1999.

GELADI, P., KOWALSKI, B.R. Partial least-squares regression: a tutorial. Analytica Chimica Acta, 185, p. 1-17, 1986.

JENKINS R., SNYDER R.L. Introduction to X-ray Powder Diffractometry. John Wiley \& Sons, Inc., 1996.

MALLEY, D. F., MARTIN, P. D., BEN-DOR, E. Application in analysis of soils. In: ROBERTS, C.A., WORKMAN, J., REEVES, J.B. (Eds.). Near-Infrared Spectroscopy in Agriculture, Soil Science Society of America, Madison, WI, p. 729-784, 2004.

MARTENS, H., NÆS, T. Multivariate calibration. John Wiley and Sons, Chichester, 1989.

NGUYEN, T.T., JANIK, L.J., RAUPACH, M., Diffuse reflectance infrared Fourier transform (DRIFT) spectroscopy in soil studies. Australian Journal of Soil Research, 29, p. 49- 67, 1991.

OLIVEIRA, F. V. C. S. R. S. de, NEUMAN, R. Quantificação de fases minerais em bauxitas por difração de raios-x e Método de Rietveld. JORNADA DE INICIAÇÃO CIENTIFICA- CETEM, 16. Anais... p. 222-228, 2009.

PÖLLMANN, U.K., ANGÉLICA, R.S. O refinamento de Rietveld como um método para o controle de qualidade de minérios de ferro. REM- Revista Escola de Minas, v. 55, n. 2, p. 111-114, 2002.

REEVES III, J.B., MCCARTY, G.W., MEISINGER, J.J. Near infrared reflectance spectroscopy for the analysis of agricultural soils. Journal of Near Infrared Spectroscopy, 7, p. 179-193, 1999.

RIETVELD, H.M., A profile refinement method for nuclear and magnetic structures. Journal of Applied Crystallography, 2, p. 65-71, 1969.

RUSSELL, J.D., FRASER, A.R. Infrared Methods. Clay Mineralogy: Spectroscopic and Chemical Determinative Methods. UK: Editora Chapman e Hall. 1994. Cap 2. p. 11-67.

SHENK, J.S., WESTERHAUS, M.O. Calibration the ISI way. In: DAVIES, A.M.C., WILLIAMS, P.C. (Eds). Near Infrared Spectroscopy: The Future Wave. Chichester, UK: NIR Publications, 1996, p. 198-202.

SPIEGELMAN, C. H., MCSHANE, M. J., COTÉ, G. L., GOETZ, M. J., MOTAMEDI, M., YUE, Q. L. Theoretical justification of wavelength selection in PLS calibration: development of a new algorithm. Analytical Chemistry, v. 70, p. 35-44, 1998.

TORRENT, J., BARRÓN, V. Diffuse Reflectance Spectroscopy. In: ULERY, A.L., DREES, L.R. (Eds.). Methods of Soil Analysis Part 5. Mineralogical Methods. Soil Science Society of America, SSSA Book Series n. 5, p. 367-387, 2008.

TORRENT, J., BARRÓN, V., Laboratory Measurement of Soil Color: Theory and Practice. In: BIGHAM J.M., CIOLKOSZ E.J. (Eds.). Soil Color, SSSA Special Publication Number 31 Madison, p. 21-33, 1993.

VISCARRA ROSSEL, R.A. PARLES: Software for chemometric analysis of spectroscopic data. Chemometrics and Intelligent Laboratory Systems, 90, 2008, p.72-83.

VISCARRA-ROSSEL, R. A., WALVOORT, D. J. J., MCBRATNEY, A. B., JANIK, L. J., SKJEMSTAD, J. O. Visible, near-infrared, mid-infrared or combined diffuse reflectance spectroscopy for simultaneous assessment of various soil properties. Geoderma, 131, p. 59-75, 2006.

Artigo recebido em 15 de março de 2010. Aprovado em 03 de fevereiro de 2011. 